Präv Gesundheitsf 2022 · 17:51-58 https://doi.org/10.1007/s11553-021-00834-z Eingegangen: 19. Oktober 2020

Angenommen: 17. Januar 2021

Online publiziert: 11. Februar 2021

(c) Der/die Autor(en) 2021

\section{Zusatzmaterial online}

Zusätzliche Informationen sind in der Online-Version dieses Artikels (https://doi. org/10.1007/s11553-021-00834-z) enthalten.

\section{Im hausärztlichen Setting sind verschiedene Möglichkeiten der Intervention denkbar, die hel- fen können, ein Fortschreiten von Demenzerkrankungen zu ver- langsamen und Lebensqualität zu bewahren [4, 7, 11, 21, 24, 29]. Hierzu gehören neben medikamen- tösen auch nicht-medikamentöse Therapieformen wie Bewegung, Physiotherapie, kognitives Training sowie eine intensive Beratung von Betroffenen und Angehörigen [8, 35]. Die vorliegende Studie basiert auf Interviews mit Hausärzt*innen aus sechs Bundesländern. Ziel war es, einen Überblick zu gewinnen, welche Ansätze und Strategien im primärärztlichen Setting ergriffen werden, um auf die Progredienz von Demenzerkrankungen einzuwirken.}

Um rechtzeitig Weichenstellungen für eine funktionierende Versorgung von Demenzerkrankten vorzunehmen, ist eine möglichst frühzeitige Diagnose entscheidend. Auch wenn es bislang an einer heilenden Therapie fehlt $[21,24,29]$, bestehen im beginnenden Stadium die güns-

Julian Wangler' · Michael Jansky

' Zentrum für Allgemeinmedizin und Geriatrie, UNIVERSITÄTSMEDIZIN Mainz, Mainz, Deutschland

Welche

\title{
Einwirkungsmöglichkeiten sehen Hausärzt*innen bei Demenzerkrankungen? - Ergebnisse einer qualitativen Studie
}

tigsten Chancen, auf das Fortschreiten der Erkrankung möglichst effektiv einzuwirken [4]. Ebenso bedeutsam ist es, für Betroffene und Angehörige so lange wie möglich eine gute Lebensqualität zu erhalten. Hierzu kommt es darauf an, beide rechtzeitig auf die Folgen der Erkrankung vorzubereiten [7, 11, 28, 29].

Aufgrund ihrer langjährigen Patientenkenntnis kommt Hausärzt ${ }^{*}$ innen bei der Erkennung demenzieller Erkrankungen große Bedeutung $\mathrm{zu}[12,20$, 25]. Da Allgemeinmediziner*innen gerade ihre älteren Patienten gut kennen, sind sie in der Lage, kognitive Veränderungsprozesse frühzeitig festzustellen $[3,15]$. Interdisziplinäre Leitlinien und Versorgungskonzepte plädieren dafür dass das hausärztliche Setting nach der Diagnosesicherung (unter fachärztlicher Mitbetreuung) eine aktive Rolle beim Krankheitsmanagement wahrnehmen sollte $[4,8]$. Aufgrund guter Kenntnisse von Lebensumständen, Befindlichkeiten und Bedürfnissen von Erkrankten und Angehörigen könne der Hilfsbedarf von Hausärzt*innen am besten identifiziert werden, sodass hier die Verantwortung für die weitere Versorgung liegen solle $[21,24,26]$.

Prinzipiell stehen im primärärztlichen Setting verschiedene Möglichkeiten zur Verfügung, dem Fortschreiten eines "mild cognitive impairment" entgegenzuwirken [13, 26]. Diverse Autoren heben die Bedeutung eines vom Hausarzt ausgehenden multimodalen, zielorientierten und dem Krankheitsverlauf anzupassenden Behandlungs- und Pflegeplans hervor, in dem auf unterschiedlichen Ebenen nicht-medikamentöse und medikamentöse Therapieformen miteinander kombiniert werden können [8]. Als medikamentöse Begleitmaßnahmen sind v.a. Antidementiva, Schlaf- und Beruhigungsmittel zu nennen [35]. Auf Seiten nicht-medikamentöser Ansätze kann von Verfahren der Bewegungsaktivierung, Physiotherapie oder kognitivem Training Gebrauch gemacht werden, um den Erhalt von Alltagsfertigkeit zu unterstützen [8]. Darüber hinaus können Hausärzt ${ }^{*}$ innen dazu beitragen, dass Patienten und Angehörige frühzeitig an Beratungs- und Hilfsangebote herangeführt werden $[7,11]$. So können optimale Voraussetzungen für einen möglichst gelingenden Alltag von Demenzpatienten in ihrem gewohnten Umfeld geschaffen, andererseits auch einem „Ausbrennen“ pflegender Angehöriger vorgebeugt werden [28]. Nationale und internationale Leitlinien sprechen sich dafür aus, jenseits medikamentöser Verfahren besonderes Augenmerk auf die bedarfsgerechte „Begleitung, Unterstützung sowie psychische und soziale Stabilisierung und Entwicklungsverzögerung der Erkrankung" zu legen [21, S. 25]. 
Die Bedeutung von trans- und intrerdisziplinären Versorgungsnetzwerken, die mit Hausärzt ${ }^{*}$ innen beim Demenzmanagement kooperieren, wird als besonders wichtig beurteilt. Trotz der teils engmaschigen Einrichtung von Demenznetzwerken und Pflegestützpunkten sowie ersten Modellprojekten [10] fehlt es im Bereich der Demenzversorgung bislang an einer breit etablierten multiprofessionellen und sektorenübergreifenden Versorgung, die ärztliche, pflegerische und weitergehende Betreuungsangebote besser verzahnen und eine möglichst klare Aufgabenteilung beinhalten, um eine bedarfsgerechte, personenzentrierte und leitlinienorientierte Versorgung von Patienten mit Demenz bieten zu können $[15,18,21]$. Das Fehlen von adäquaten und wirksamen Strukturen zur ambulanten Krisenintervention führt i.d.R. bei Krisensituationen rasch zu Krankenhauseinweisungen, die nicht nur mit höheren Kosten einhergehen, sondern auch Komplikationen für die Patienten mit sich bringen können [33].

Mit Blick auf das hausärztliche Management wird moniert, dass „die Behandlungsrealität hinter dem Soll-Zustand der Leitlinien zurückliegt" [21, S. 24]. Wie Studien gezeigt haben, hängt dies zusammen mit dem Umstand, dass Demenzdiagnosen in der Hausarztpraxis oft nicht oder erst zu einem späten Zeitpunkt gestellt werden $[6,17,31]$. Auch wurde festgestellt, dass die hausärztliche Kenntnis von demenzbezogenen Diagnostik- und Versorgungsoptionen oft nicht in ausreichendem Maß gegeben ist $[1,2,13,15,24,27,30,35]$. Die Bereitschaft, Demenzpatienten therapeutisch $\mathrm{zu}$ begleiten, ist nur bei einem Teil der Hausärzt*innen vorhanden [32]. Als ursächlich für diese Reserviertheit werden Faktoren wie Zeitdruck und Ressourcenknappheit gesehen, aber auch geringe Wirksamkeitserwartungen aufgrund eines erlebten Mangels an Therapieoptionen $[13,14,27]$. Ferner werden die enge Beziehung des Hausarztes zum Patienten und der Anspruch, diesen zu kennen, als Ursachen einer verschleppten Therapie angeführt [3, 26, 34].

Jenseits der oben angesprochenen $\mathrm{Be}$ funde früherer Arbeiten sind für den deutschsprachigen Raum bislang nur we- nige belastbare Studien vorgelegt worden, die auf Grundlage einer explorierenden Vorgehensweise der Frage nachgehen, welche therapeutischen Maßnahmen Hausärzt ${ }^{*}$ innen bevorzugt ergreifen, um auf Demenzerkrankungen einzuwirken und wie ihre Erfahrungen beim Management ausfallen [30, 32]. Die vorliegende Arbeit soll einen Beitrag leisten, hausärztliche Einstellungen und Erfahrungen zu explorieren, wenn es darum geht, bereits festgestellten Demenzerkrankungen entgegenzuwirken.

\section{Methodik}

\section{Studienkonzeption}

Da beim betrachteten Thema ein Mangel an Studien zu konstatieren ist, besteht Bedarf nach einer breiteren Exploration. Entsprechend fiel die Wahl auf qualitative, halbstandardisierte Leitfadeninterviews mit Hausärzt*innen. Zum einen konnte das Thema so möglichst unvoreingenommen nach neuen Aspekten und Zusammenhängen sondiert werden, zum anderen gibt eine Interviewstudie Hausärzt*innen die Möglichkeit, ihre eigenen Standpunkte und Erfahrungen ausführlich darzustellen.

Das Erkenntnisinteresse lässt sich anhand folgender Fragestellungen beschreiben:

- Inwiefern sind Hausärzt*innen in das therapeutische Management von Demenzpatienten involviert?

- Welche Interventionsansätze werden als effektiv erachtet, um dem Fortschreiten von Demenzerkrankungen entgegenzuwirken?

- Welche Herausforderungen werden beim Management von demenziell Erkrankten wahrgenommen?

- Welche Verbesserungsansätze werden als sinnvoll erachtet, um die Dimension der Demenzprävention zu fördern?

Die Studie fokussiert auf ein beginnendes bis mittleres Demenzstadium, weil hier die Chancen einer positiven Einwirkung durch gezielte intervenierende Maßnahmen als vergleichsweise groß anzusehen sind [4].

\section{Leitfaden}

Entlang der Forschungsfragen wurde ein kompakter Leitfaden erstellt (s. Anhang). Der Leitfaden wurde im $\mathrm{Zu}$ ge einer Literaturrecherche sowie auf Grundlage mehrerer von den Autoren durchgeführter Vorstudien erstellt, deren Schwerpunkt auf hausärztlichen Einstellungen, Handlungsmustern und Kompetenzindikatoren in Bezug auf die Demenzdiagnostik liegt [30-32]. Prominente Berücksichtigung bei der Leitfadenerstellung erfuhr zudem die Arbeit von Radisch et al. [21], die ein Konzept für eine integrierte, hausarztbasierte Demenzversorgung vorgelegt haben. Eine weitere Spezifizierung des Leitfadens erfolgte im Zuge der ersten Interviews.

Das Instrument besteht aus 22 übergeordneten Fragestellungen mit verschiedenen Unterfragen. Bei den Befragungsdimensionen handelte es sich um: Rollenverständnis bei der Demenzversorgung, wahrgenommene Einwirkungsmöglichkeiten auf Demenzerkrankungen im hausärztlichen Setting, praktizierte Interventionsansätze und Erfahrungen, erlebte Herausforderungen beim (therapeutischen) Management, Bedeutung von und Kooperation mit regionalen Hilfs- und Unterstützungsnetzwerken, Optimierungsansätze in Bezug auf eine hausärztliche Demenzprävention, Einschätzung subjektiver Kompetenz und Vorerfahrung in Bezug auf die Thematik (s. Anhang). Eigene Positionen oder Vorerfahrungen flossen nicht ein.

\section{Rekrutierung, Sample und Durchführung}

Die zu befragenden Hausärzt ${ }^{*}$ innen haben ihren Praxisstandort in BadenWürttemberg, Hessen, Nordrhein-Westfalen, Rheinland-Pfalz, Saarland und Thüringen. In Anbetracht eines qualitativ-explorativen Vorgehens wurde eine begrenzte Zahl von Praxen in den genannten Bundesländern systematisch rekrutiert.

In einem ersten Schritt wurde ein Pool von insgesamt 84 potenziellen Kontaktadressen erstellt, der eine große Bandbreite von hausärztlichen Praxen in 
Präv Gesundheitsf 2022 · 17:51-58 https://doi.org/10.1007/s11553-021-00834-z

(c) Der/die Autor(en) 2021

\section{J. Wangler $\cdot$ M. Jansky}

\section{Welche Einwirkungsmöglichkeiten sehen Hausärzt*innen bei Demenzerkrankungen? - Ergebnisse einer qualitativen Studie}

\section{Zusammenfassung}

Hintergrund. Obwohl es an einer heilenden Therapie für Demenzerkrankungen fehlt, bestehen im hausärztlichen Setting diverse Möglichkeiten, dem Fortschreiten eines "mild cognitive impairment" entgegenzuwirken. Bislang sind nur wenige Studien vorgelegt worden, die der Frage nachgehen, welche therapeutischen Strategien Hausärzt*innen bevorzugt ergreifen, um auf Demenzerkrankungen einzuwirken.

Ziel der Arbeit. Ermittelt wird, welche Interventionsansätze für das Management von Demenzpatienten als effektiv erachtet werden, sowie erlebte Herausforderungen und gewünschte Optimierungsmaßnahmen zur Stärkung der Demenzprävention. Material und Methoden. Zwischen März und September 2020 wurden 42 halbstandardisierte Interviews mit Hausärzt ${ }^{*}$ innen in Baden-Württemberg, Hessen, NordrheinWestfalen, Rheinland-Pfalz, Saarland und Thüringen geführt.

Ergebnisse. Viele Interviewte schätzen die Bedeutung einer Demenzprävention als hoch ein und gehen davon aus, einen wirksamen Beitrag leisten zu können - nicht-medikamentösen Ansätzen wird großer Stellenwert eingeräumt. Die Lotsenfunktion des Hausarztes wird gezielt eingesetzt, um Patienten und Angehörige an Beratungs- und Hilfsakteure heranzuführen. Ein Teil des Samples sieht nur medikamentöse Therapieformen als geeignet an, um Einfluss auf Demenzerkrankungen zu nehmen. In dieser Gruppe sind geringe Selbstwirksamkeitserwartungen verbreitet. Als häufige Herausforderungen werden im Sample Probleme in der interdisziplinären Zusammenarbeit artikuliert, die nicht selten einem kohärenten Therapiekonzept entgegenstehen.

Diskussion. Hausärzt*innen sollten bestärkt werden, die Priorisierung der primären Behandlungsstrategie bei der Demenzversorgung zu übernehmen. Wichtig erscheint, dass auch die Potenziale nicht-medikamentöser Interventionen wahrgenommen werden. Die Zusammenarbeit zwischen Hausärzt ${ }^{*}$ innen und Hilfsnetzwerken sollte gestärkt werden. Zudem sollte die Entwicklung integrierter Versorgungskonzepte und strukturierter Behandlungsprogramme vorangetrieben werden.

\section{Schlüsselwörter}

Demenzversorgung $\cdot$ Hausarzt · Demenzdiagnostik · Intervention - Therapie . Management

\section{What intervention options do general practitioners recommend in the case of dementia?-Results of a qualitative study}

\section{Abstract}

Background. Although there is currently no curative therapy for dementia, there are many options in the general practitioner (GP) setting to counteract the progression of mild cognitive impairment and, thus, to pursue prevention. To date, only a few studies have investigated which therapeutic strategies GPs prefer to use to influence dementia.

Objectives. In the course of the study, it will be determined which intervention approaches GPs consider to be effective for the management of dementia patients, which challenges they are experiencing and which measures they would like to take to strengthen the dimension of dementia prevention.

Materials and methods. Between March and September 2020, 42 semistandardized interviews were conducted with GPs in BadenWürttemberg, Hesse, North Rhine-Westphalia, Rhineland-Palatinate, Saarland and Thuringia. Results. Many interviewees rate the importance of prevention of dementia as high and assume that they can make an effective contribution; great importance is attached to nondrug approaches. The guiding function of the family doctor is used in a targeted manner to introduce patients and relatives to counseling and aid services. It is noticeable that a portion of the physicians only identify intervention options in drug therapy. Lower expectations are common in this group. Problems in interdisciplinary cooperation are addressed as frequent challenges in the sample, which often conflict with a coherent therapy concept.
Discussion. General practitioners should be encouraged to prioritize the primary treatment strategy in dementia care. It is also important that the potential of nondrug interventions be recognized. Cooperation between general practitioners and support networks should be strengthened. In addition, the development of integrated care concepts and structured treatment programs should be promoted.

\section{Keywords}

Dementia care - General practitioner . Dementia diagnostics · Intervention · Therapy . Management den genannten Bundesländern enthielt. Daraufhin erfolgte die Rekrutierung des Samples. Um sicherzustellen, dass bestimmte Merkmale (Geschlecht, Niederlassungsform, Praxisumgebung) im Sample annähernd gleichwertig verteilt sind, wurde für diese mit einer vordefinierten Quotierung gearbeitet. Zudem wurde Wert auf eine möglichst breite geografische Verteilung der Praxen in den einzelnen Bundesländern gelegt sowie auf die Berücksichtigung unterschiedlicher Altersgruppen und Weiterbildungshintergründe. So konnte ein heterogenes Sample gewonnen werden.

Insgesamt wurden 59 Hausärzt ${ }^{*}$ innen in den Bundesländern Baden-Würt- temberg, Hessen, Nordrhein-Westfalen, Rheinland-Pfalz, Saarland und Thüringen kontaktiert, wobei 42 Interviews geführt wurden (7 Ärzte pro Bundesland). - Tab. 1 zeigt das letztendlich gewonnene Sample.

Nach der Gewinnung der Stichprobe erfolgte die Durchführung der Studie. Im Vorfeld erhielten die Interviewten ei- 


\section{Originalarbeit}

\begin{tabular}{|c|c|}
\hline Alter & $\emptyset 52$ Jahre \\
\hline Geschlecht & 23 männlich, 19 weiblich \\
\hline Niederlassungsform & 20 Gemeinschaftspraxis, 22 Einzelpraxis \\
\hline Praxisumgebung & 17 Landgemeinde/Kleinstadt, 12 Mittelstadt, 13 Großstadt \\
\hline Status & 30 Praxisinhaber, 12 angestellte Ärzte \\
\hline $\begin{array}{l}\text { Weiterbildungs- } \\
\text { hintergrund }\end{array}$ & $\begin{array}{l}13 \text { themenbezogene Fortbildungen, } 7 \text { regelmäßige Teilnahme an Qualitäts- } \\
\text { zirkeln, } 6 \text { geriatrische Weiterbildung }\end{array}$ \\
\hline
\end{tabular}

ne Aufklärung über das Gesprächsthema sowie eine schriftliche Einverständniserklärung. Die Interviews fanden zwischen März und September 2020 statt und wurden in 29 von 42 Fällen telefonisch durchgeführt (40 bis $70 \mathrm{~min}$ ).

\section{Auswertung}

Die theoretische Sättigung wurde erreicht, sodass auf weitere Interviews verzichtet werden konnte. Von theoretischer Sättigung wird in der qualitativen Forschung gesprochen, wenn die Erhebung weiterer Daten und deren Analyse keine neue Aspekte an einer Kategorie oder einem Kategoriensystem und somit keine neuen Erkenntnisse mehr zutage fördert. Dies zeichnete sich im Zuge der zuletzt geführten Interviews ab.

Die im Anschluss an die Datenerhebung erstellten Transkripte wurden im Team mithilfe einer qualitativen Inhaltsanalyse nach Mayring [16] mit der Software MAXQDA (VERBI, Berlin) ausgewertet. Zunächst wurden die bedeutungstragenden Grundaussagen herausgearbeitet, anschließend weiter abstrahiert und zusammengefasst und schließlich ein Kategoriensystem erstellt, das sich eng am Leitfaden orientierte und mit Fortgang der Auswertung wiederholt geprüft und ggf. modifiziert wurde. Im Mittelpunkt stand, die unterschiedlichen Sichtweisen und Erfahrungen logisch zu kategorisieren.

\section{Ergebnisse}

\section{Einstellung und Involvierung bezüglich Demenzprävention}

Es geben 38 Interviewte an, in der eigenen Praxis eine Demenzdiagnostik vorzuhalten; 35 bekunden, häufiger oder gelegentlich in das therapeutische Manage- ment von Demenzerkrankten involviert zu sein, wobei 12 Ärzte betonen, sich hier stärker nach dem Vorgehen des zuständigen Facharztes zu richten. Unter den Befragten ist das Spektrum an Auffassungen über Sinn und Nutzen einer Demenztherapie groß.

„Natürlich müssen wir alles unternehmen, um Einfluss auf Degenerationsprozesse $z u$ nehmen." (I-8m)

"Sie können da nichts aufhalten. Das Kind ist ab diesem Zeitpunkt schon in den Brunnen gefallen." (I-1m)

Rund die Hälfte der Befragten $(n=20)$ vertritt mit Blick auf die Demenzprävention reservierte bis skeptische Auffassungen. Es fällt auf, dass die Ursachen hierfür verbreitet in negativen (Selbst-)Wirksamkeitsüberzeugungen liegen. So hebt eine Interviewte hervor, wer eine Therapie von Demenzerkrankten anstrebe, der betreibe „nicht mehr als Mangelverwaltung" (I-33w). Andere Befragte fokussieren die eigenen Ohnmachtsempfindungen:

Du setzt Dich als Arzt einem Gefühl aus, nicht viel tun zu können. (I-2m)

\section{Maßnahmen zur Demenz- prävention und beobachtete Effekte}

Das Gros der Ärzte $(n=37)$ betont, dass ein Beibehalten des Patienten im häuslichen Umfeld das Ziel sein und die Einweisung ins Krankenhaus oder Pflegeheim möglichst verhindert werden müsse.

Unter den Gesprächspartner*innen, die sich an der Therapie beteiligen $(n=$ 35), fallen zwei Gruppen auf. 13 Befragte betrachten Maßnahmen der Prävention ausschließlich unter medikamentösen Gesichtspunkten.
Ich wüsste nicht, was es da groß an Optionen gäbe, außer die bestehenden Präparate zu verschreiben, also Dinge wie Antidementiva, Psychopharmaka und so weiter. (I-1m)

Die übrigen 22 Befragten gehen deutlich darüber hinaus, indem sie die medikamentöse Begleitung mit anderen Maßnahmen kombinieren oder auch die Medikation gegenüber anderen Therapieformen zurückstellen.

In den Interviews wurde eine große Bandbreite an flankierenden Maßnahmen ersichtlich (vgl. @ Tab. 2). Dabei sind die entsprechenden Hausärzt ${ }^{*}$ innen der Überzeugung, dass Faktoren wie intrinsische Motivation und Kreativität, gezieltes Wahrnehmungs- und Aufmerksamkeitstraining, aber auch Entspannungstechniken und Bewegungsförderung Einflussfaktoren darstellen, um das Fortschreiten einer Demenzerkrankung auszubremsen. Diese integrativ agierenden Ärzte gehen davon aus, dass man Demenz „nur im dynamischen $\mathrm{Zu}$ sammenhang wirksam aufhalten kann, [...] also unter Einbeziehung von physischen, kognitiven und psychosozialen Verfahren “ (I-4w). Großer Wert wird zudem angehörigenbezogenen Interventionen beigemessen.

Vor diesem Hintergrund sehen sich besagte Ärzt ${ }^{*}$ innen als Schnittstelle zu Hilfs- und Unterstützungsakteuren; teilweise wird auch in kommunalen Netzwerkstrukturen agiert. Es bestehen oft intensive Kooperationen mit Pflegestützpunkten, Pflegediensten, (Physio)therapeuten, Gesundheitszentren und Selbsthilfegruppen.

Die Befragten berichten von ihren Beobachtungen, wie der „resignative und potenziell depressionsfördernde Impact einer Demenzdiagnose" durch eine geschickte Kombination nicht-medikamentöser Maßnahmen „längerfristig mehr als nur kompensiert werden kann" (I-32m). Entscheidend sei, dass sowohl Patienten als auch Angehörige "unter den neuen Bedingungen für sich ein neues Gleichgewicht" finden (I-13w). Hausärzt ${ }^{\star}$ innen könnten bedarfsnah helfen, „einen Zugang zu Hilfsstrukturen zu bahnen “ (I-22m) mit dem Ziel, dass „Belastungen verringert, Lebens- 


\begin{tabular}{|c|c|c|}
\hline Präferierte Maßnahme & Beispielzitat & $\begin{array}{l}\text { Anzahl } \\
\text { Befragte }\end{array}$ \\
\hline Systematische Therapie von Komorbiditäten & $\begin{array}{l}\text { "Sie müssen Risikomanagement betreiben und sollten Dinge wie Delir, Herzin- } \\
\text { suffizienz, Diabetes konsequent behandeln, da es die Demenzsymptome klar }\end{array}$ & 18 \\
\hline $\begin{array}{l}\text { Körperliche Aktivierung: Bewegung/Sport, Physio- } \\
\text { therapie/Ergotherapie }\end{array}$ & $\begin{array}{l}\text { "Die Fähigkeit, körperliche Alltagsfunktionen aufrechtzuerhalten, geht Hand in } \\
\text { Hand mit der kognitiven Funktion. [...] Die Leute sind länger frisch." (I-15w) }\end{array}$ & 8 \\
\hline Kognitives Training, kognitive Stimulation & $\begin{array}{l}\text { "Wenn Sie die Wahrnehmung, Aufmerksamkeit und Erinnerung systematisch } \\
\text { trainieren, hat das einen großen Nutzen." (I-18m) }\end{array}$ & 9 \\
\hline (Künstlerisch )kreative Therapien & $\begin{array}{l}\text { "Wenn Sie mich fragen, ist das Beste, die Kreativität in den Leuten anzusprechen. } \\
\text { Machen Sie Musik, irgendetwas Künstlerisches oder tanzen Sie." (I-4w) }\end{array}$ & 5 \\
\hline $\begin{array}{l}\text { Psychosoziale Unterstützung, psychoedukative } \\
\text { Intervention, psychiatrische Behandlung }\end{array}$ & $\begin{array}{l}\text { "Sie können Patienten durch Trainings beibringen, einen positiven Umgang mit } \\
\text { der Erkrankung zu erlernen. Diese innere Ausgeglichenheit spielt eine große Rolle." }\end{array}$ & 7 \\
\hline $\begin{array}{l}\text { Angehörigenbezogene Interventionen: Verhal- } \\
\text { tensmanagement, Bewältigungstherapien, Entlas- } \\
\text { tungsmöglichkeiten }\end{array}$ & $\begin{array}{l}\text { "Die Angehörigen sind oft die eigentliche Einflussgröße. Sie müssen alles daran } \\
\text { setzen, diese Personen in ihrer Resilienzfähigkeit zu stärken. Daran hängt auch } \\
\text { der Patient." (I-16w) }\end{array}$ & 15 \\
\hline
\end{tabular}

qualität erhalten und im subjektiven Empfinden die Chancen betont werden“ (I-14w). Hätten Demenzbetroffene und Angehörige "Zutrauen in den eigenen Alltag“, sei auch „mehr Ausgeglichenheit und Stabilität" die Folge (I-14w). Dies sei der eigenen Einschätzung nach „mit der wichtigste Indikator für einen günstigeren Verlauf einer Demenz" (I39w).

Ein Großteil des Samples betont, dass eine objektive Wirksamkeitsüberprüfung therapeutischer Maßnahmen bei Demenzerkrankungen schwierig sei. Dennoch geben sich viele Interviewte überzeugt, dass es diese Wirkung „stets mit sehr individueller Ausprägung gibt [...] und damit auch so etwas wie echte Sekundärprävention“ (I-12m).

\section{Erlebte Herausforderungen}

Mit Blick auf wahrgenommene Hürden und Herausforderungen bei der Demenzprävention spricht ein Teil der Hausärzt ${ }^{\star}$ innen die Problematik an, keinen ausreichenden Überblick zu besitzen, welche Gesundheits- und Versorgungsakteure Demenzbetroffenen und Angehörigen beratend weiterhelfen können. Dies sind v.a. Interviewte aus der Gruppe, die einen Einsatz nicht-medikamentöser Maßnahmen nicht erwägen.

Zudem werden die Punkte Medikation und interdisziplinäre Zusammenarbeit des Öfteren angeführt, nicht selten in einem direkten Zusammenhang. Ein Teil der Ärzt ${ }^{*}$ innen $(n=19)$ hinterfragt , in einer sehr prinzipiellen Weise" (I-33w) den
Nutzen etablierter Demenzmedikamente aufgrund eigener Erfahrungswerte.

Ich habe in den letzten 20 Jahren einen Haufen Patienten gesehen, die auf diesen ganzen Psychopharmaka- oder Antidementivamitteln unterwegs sind - da war nichts dabei, das vielversprechend gewesen wäre. (I-15w)

Auffällig ist, dass es insbesondere unter Befragten, die bei der Therapie in erster Linie auf Medikamente abstellen, eine große Unzufriedenheit mit den Effekten der Behandlung gibt.

Man kann sicher etwas als Therapie anvisieren, aber es wäre falsch, Erwartungen oder Hoffnungen zu haben, weil man denen ohnehin nicht gerecht wird. (I-9m)

Einige Befragte berichten von Nebenwirkungen wie Persönlichkeitsveränderungen (Gemütsschwankungen, Zerrüttung des Tag-Nacht-Rhythmus) aufgrund der Einnahme von Neuroleptika, sodass man beim Verordnen medikamentöser Maßnahmen „sehr vorsichtig geworden“ geworden sei (I-15w).

Unter einem Großteil aller in therapeutische Maßnahmen involvierten Ärzte $(n=24)$ wird Kritik an der interdisziplinären Zusammenarbeit artikuliert. Jenseits der mangelnden Verfügbarkeit psychiatrischer oder neurologischer Fachärzte und langer Wartezeiten wird eine fachärztliche Tendenz beschrieben, hausärztliche Handlungsspielräume einzuschränken, indem ohne Abstimmung eigenmächtige und oft nicht nachvollziehbare Verordnungen erfolgten.
Oft geht es mir so: Wieso jetzt dieses Medikament? Der Psychiater gibt sein Urteil $a b$, und wir Hausärzte sind dann die Befehlsempfänger und werden auf einen Therapiepfad gelenkt, den wir so nicht eingeschlagen hätten. (I-28m)

In einigen Fällen wird von „fatalen Konsequenzen“ durch „übertriebene Medikamente und Dosierungen“" (I-12m) berichtet, die zu den angesprochenen Negativfolgen für die psychische Integrität führten. Unter den Befragten, die nicht-medikamentöse Maßnahmen und das Ziel der psychosozialen Stabilisierung stärker verfolgen, wird die fachärztliche Medikationsstrategie z. T. im Konflikt und Widerspruch zum eigenen Vorgehen erlebt.

Man hat manchmal dieses Gefühl, dass da das eigene Bemühen, den Patienten aufgebaut zu bekommen, wieder konterkariert wird. (I-10m)

Einige Befragte äußern sich aber auch selbstkritisch und räumen ein, dass viele Hausärzt ${ }^{*}$ innen nicht immer im ausreichenden Maß den Austausch mit fachärztlichen Kollegen suchten.

Wir sind alle als Einzelkämpfer unterwegs. Damit kommt man bei Demenz, wo im Konzert an so vielen Stellschrauben gedreht werden muss, kaum weiter. (I$34 w)$

Als weitere bei der Demenzprävention relevante Problematik wird die Belastung des Arzt-Patient-Verhältnisses infolge der Diagnosestellung angesprochen. Der Umgang mit bestimmten Patienten 
sei häufig schwierig, da diese sich den geistigen Abbau aus Angst vor einem Verlust der Entscheidungsautonomie nicht eingestehen wollten, sodass auch die Compliance leide.

\section{Optimierungsansätze}

Auf die Frage, welche Verbesserungen ergriffen werden sollten, um die Dimension der Prävention von Demenzerkrankungen in der Hausarztpraxis $\mathrm{zu}$ stärken, brachten die Interviewten mehrere Punkte wiederholt vor. Stark moniert $(n=14)$ wurde das Fehlen strukturierter, sektorenübergreifender Behandlungsprogramme mit Verankerung in der Primärversorgung.

Warum gibt es kein Disease-Management-Programm Demenz? Was wir brauchen, sind Programme, die das Interdisziplinäre und den Multimethodenansatz stärken. (I-41w)

Zudem regten mehrere Befragte $(n=9)$ die Entwicklung eines evidenzbasierten, hausarztkonformen Diagnose- und Therapiealgorithmus für den Umgang mit Demenzpatienten an. Dieser würde nicht nur dabei helfen, Symptome zielgerichtet abzuklären, sondern ein besseres Verständnis ermöglichen, wie therapeutische Schritte zu gestalten sind. Insbesondere könne ein in der Breite etablierter und akzeptierter Algorithmus wirksam dazu beitragen, dass „Hausund Fachärzte an einem Strang ziehen“ (I-3m).

Einige Befragte $(n=5)$ sehen die hausärztliche Rolle beim Krankheitsbild selbstkritisch und betonen, dass Allgemeinärzt ${ }^{\star}$ innen mehr Zeit und Energie in gezielte Weiterbildungen sowie in die Bereitschaft, Demenzpatienten konsequent $\mathrm{zu}$ diagnostizieren und $\mathrm{zu}$ betreuen, investieren sollten.

Nur, wenn wir es selbst in der Hand behalten, können wir das machen, was wir für das Beste halten. Das setzt aber voraus, dass Hausärzte sich in der ganzen Breite mit dem Thema beschäftigen. (I-34w)

\section{Diskussion}

\section{Zusammenfassung und Befunde anderer Studien}

Die Interviews zeigen, dass ein beträchtlicher Teil der Hausärzt ${ }^{*}$ innen die Bedeutung einer Demenzprävention als hoch einschätzt und davon ausgeht, durch eigene Interventionen einen wirksamen Beitrag leisten zu können. Zudem räumen viele Allgemeinärzte (integrativen) therapeutischen Maßnahmen jenseits von medikamentösen Behandlungsansätzen einen beachtlichen Stellenwert ein. In diesem Zusammenhang haben die Interviews ein breites Spektrum an Therapiestrategien offengelegt. Dabei wird die Lotsenfunktion des Hausarztes gezielt eingesetzt, um Patienten und Angehörige bedarfsorientiert an Beratungs- und Hilfsakteure heranzuführen und so die psychosoziale Stabilität zu unterstützen.

Auf der anderen Seite fällt ein Teil der Befragten auf, der die Möglichkeiten, Einfluss auf die Progredienz von Demenzerkrankungen auszuüben, ausschließlich in medikamentösen Therapieformen ausmacht. Gerade in dieser Gruppe sind geringe Selbstwirksamkeitserwartungen verbreitet, weil die eigenen Behandlungserfahrungen oft nicht zufriedenstellend ausgefallen sind. Auch besteht eine Tendenz, Fachärzten die therapeutische Steuerung zu überlassen und sich aus einer aktiven Patientenbegleitung zurückzuziehen. Damit einhergehend, fällt in dieser Gruppe der Interviewten eine nicht immer ausreichende Kenntnis in Bezug auf die Thematik der Demenzversorgung auf.

Als Herausforderungen beim Hinwirken auf eine Prävention von Demenzerkrankungen werden im Sample beim Einsatz von Medikamenten negative Effekte auf die seelische Verfassung und Persönlichkeitsstruktur beschrieben; zudem werden Probleme in der interdisziplinären Zusammenarbeit artikuliert, die nicht selten einem kohärenten Therapiekonzept entgegenstehen.

Insgesamt bestätigen die Interviews nicht nur viele Ergebnisse der Vorstudien [30-32], sondern fügen sich in die Befundlage, der zufolge trotz der günstigen
Position von Hausärzt ${ }^{*}$ innen, kognitive Veränderungen von Patienten rechtzeitig zu erkennen und die Therapie zu koordinieren, vielfältige Hindernisse bestehen [11]. Frühere Arbeiten deuten darauf hin, dass Allgemeinmediziner bei der Demenzversorgung eine mangelnde therapeutische Konsequenz aufgrund einer Konzentration auf rein medikamentöse Verfahren wahrnehmen; nicht-medikamentöse Verfahren finden nur begrenzt Beachtung [13-15, 21, 31, 34]. Zudem zeigen Studien, dass Hausärzt*innen nicht immer in die Behandlung von Demenzerkrankungen involviert sein möchten, weil sie Unsicherheiten im diagnostischen und therapeutischen Ablauf verspüren und nicht immer über das notwendige Hintergrund- bzw. Anwendungswissen verfügen $[2,5,14,27,30]$. Hinzu kommen in Teilen als spannungsreich erlebte Rollenkonstellationen im Umgang mit Patienten [19, 30]. Ebenfalls ist festgestellt worden, dass nur eine Gruppe von Hausärzt*innen Patienten und Angehörige an Hilfseinrichtungen (z. B. Pflegestützpunkte, Demenznetzwerke) vermittelt, da Kenntnisse über solche Akteure oft nicht vorhanden sind $[28,31]$.

Eine Reihe von Forschungsarbeiten betont die Bedeutung nicht-medikamentöser Interventionen, die frühzeitige Heranführung von Betroffenen und Bezugspersonen an Hilfsakteure sowie den herausragenden Wert einer (präventiven) Stabilisierung pflegender Angehöriger [7, 21, 28, 29]. Nach Kurz et al. [9] kann so erreicht werden, dass Erkrankte sich ausgeglichen fühlen und in ihrer Alltagsbewältigung abgestützt werden. Hirsch [8] spricht sich für einen multimodalen Therapieansatz aus ergo- und körperorientierter Therapie sowie aktivierenden Pflegemaßnahmen und ggf. psychotherapeutischen Interventionen aus. Demgegenüber sprechen sich Autoren wie Romero [23] und Reuster et al. [22] für eine gezielte und frühzeitige Parallelanwendung nicht-medikamentöser und medikamentöser Verfahren vor dem Hintergrund eines möglichst ganzheitlichen Blicks auf Patient und Angehörige aus. Hierzu sollte auch in der Aus- und Weiterbildung verstärkt Wissen vermittelt werden. 
Damit korrespondierend, artikulieren die Interviewten den Wunsch nach Versorgungsstrukturen zur systematischen Therapie von Demenzerkrankten. Dies findet seine Entsprechung in diversen Expertisen [21]. Jenseits der individuellen hausärztlichen Arbeit besteht ein Mangel an Behandlungs- und Interaktionsstrukturen, die eine multiprofessionelle, sektorenübergreifende Versorgung ermöglichen, um eine bedarfsgerechte und leitlinienorientierte Versorgung von Patienten mit Demenz bieten zu können [14, 18]. Das Fehlen von wirksamen Strukturen zur ambulanten Krisenintervention führt i.d.R. bei Krisensituationen rasch zu Krankenhauseinweisungen, die Komplikationen mit sich bringen können [33].

\section{Stärken und Schwächen}

Die Studie weist trotz des heterogenen, verschiedene Bundesländer umfassenden Samples verschiedene Limitationen auf, die entsprechend zu reflektieren sind. Neben der begrenzten Fallzahl handelt es sich um regionale Rekrutierungsschwerpunkte. Hinsichtlich der Mitwirkungsbereitschaft der Gesprächspartner ${ }^{\star}$ innen sollte berücksichtigt werden, dass verstärkt Hausärzt*innen teilgenommen haben könnten, bei denen ein thematisches Interesse vorliegt. Ferner wurde ein großer Teil der Interviews telefonisch geführt, was gegenüber Face-toface-Befragungen Einschränkungen für die Aussagekraft der Ergebnisse mit sich gebracht haben kann.

Die Studienergebnisse lassen sich einsetzen, um vertiefende Befragungen einer größeren Zahl von Hausärzt ${ }^{*}$ innen durchzuführen (z.B. deutschlandweiter Repräsentativsurvey), sodass Einstellungs- und Handlungsmuster mit Blick auf Einwirkungsmöglichkeiten im Primärarztsetting genauer beleuchtet werden können; ggf. könnten die Ergebnisse auch im Rahmen von Fokusgruppendiskussionen eingespeist werden, um wahrgenommene Problematiken und Verhaltensweisen bei der Demenzversorgung deutlicher zu erschließen. $\mathrm{Zu}$ dem lassen sich die Befunde der Arbeit für die Entwicklung bedarfsorientierter, anwendungsnaher Trainings oder Weiterbildungsformate nutzen.

\section{Schlussfolgerungen}

Folgende Ansatzpunkte für eine Optimierung der primärärztlichen Demenzprävention lassen sich vor dem Hintergrund der Befunde ableiten:

- Hausärzt*innen sollten bestärkt werden, die Priorisierung der primären Behandlungsstrategie bei der Versorgung von Demenzpatienten sowie koordinierende Funktionen zu übernehmen.

- Wichtig erscheint für die hausärztliche Arbeit, dass Einflussmöglichkeiten im Feld der Demenzversorgung nicht nur hinsichtlich medikamentöser Interventionen gesehen werden, sondern gerade nicht-medikamentöse Ansätze die Aufrechterhaltung einer guten Lebensqualität sicherstellen können. Angesichts nicht immer ausreichender Wissensbestände in Bezug auf die Versorgung Demenzerkrankter wäre es sinnvoll, diesen Zusammenhang auch über eine verstärkte Behandlung im Rahmen der ärztlichen Ausbildung gezielter zu verankern.

- Indem Patienten und Angehörige rechtzeitig an regionale Beratungsund Hilfsnetze herangeführt werden, kann ein entscheidender Beitrag zu gelingender Pflege und psychosozialer Stabilisierung geleistet werden. Insgesamt sollte die Zusammenarbeit zwischen Hausärzt ${ }^{*}$ innen und solchen Versorgungsakteuren gestärkt werden.

- Als Schlüssel zu effektiven Interventionen im Bereich der Demenzversorgung erscheinen integrierte Versorgungskonzepte und strukturierte Behandlungsprogramme, an denen es jedoch bislang mangelt.

- Ein stärker hausarztkonformer Diagnose- und Therapiealgorithmus für den Umgang mit Demenzpatienten würde helfen, eine zielgerichtete $\mathrm{Ab}$ klärung von Symptomen zu leisten. Auch könnte er helfen, die Zusammenarbeit mit Fachärzten effektiver zu strukturieren.

- Gezielte (geriatrische) Weiterbildungen und Trainings tragen dazu bei, die Sensitivität in Bezug auf Demenzdiagnostik und -therapie zu erhöhen sowie die Wissens- und Kompetenzdimension von Hausärzt*innen im Themenzusammenhang zu stärken. Insofern sollten Möglichkeiten erwogen werden, Weiterbildungen wie die Fachkunde Geriatrie für Hausärzt*innen attraktiver zu gestalten.

\section{Fazit für die Praxis}

\section{- Das hausärztlichen Setting ist für die konsequente Erkennung und das Management demenzieller Erkrankungen entscheidend. \\ - Ein erheblicher Teil der befragten Hausärzt*innen schätzt die Bedeu- tung einer Prävention von Demenz- erkrankungen als hoch ein und geht davon aus, durch eigene (nicht-me- dikamentöse) Interventionen einen wirksamen Beitrag leisten zu können. \\ - Insgesamt sollte die Zusammenarbeit zwischen Hausärzt*innen und ande- ren Versorgungsakteuren ausgebaut werden. Wichtig ist die rechtzeitige Heranführung von Patienten und Angehörige an regionale Hilfsnetze, um Pflegesettings zu stabilisieren. \\ - Als Schlüssel zu effektiven Interven- tionen im Bereich der Demenzver- sorgung und -prävention erscheinen integrierte Versorgungskonzepte und strukturierte Behandlungspro- gramme.}

\section{Korrespondenzadresse}

\section{Dr. Julian Wangler}

Zentrum für Allgemeinmedizin und Geriatrie, UNIVERSITÄTSMEDIZIN Mainz

Am Pulverturm 13, 55131 Mainz, Deutschland Julian.Wangler@unimedizin-mainz.de

Funding. Open Access funding enabled and organized by Projekt DEAL.

\section{Einhaltung ethischer Richtlinien}

Interessenkonflikt. J. Wangler und M. Jansky geben an, dass kein Interessenkonflikt besteht.

Für diesen Beitrag wurden von den Autoren keine Studien an Menschen oder Tieren durchgeführt. Für die aufgeführten Studien gelten die jeweils dort angegebenen ethischen Richtlinien. Bei der vorliegenden Studie wurden keinerlei sensible Patientendaten erhoben oder klinische Tests durchgeführt. Eine Rückversi- 
cherung bei der Ethikkommission des Bundeslandes Rheinland-Pfalz ergab, dass ein Ethikvotum nicht erforderlich ist.

Open Access. Dieser Artikel wird unter der Creative Commons Namensnennung 4.0 International Lizenz veröffentlicht, welche die Nutzung, Vervielfältigung, Bearbeitung, Verbreitung und Wiedergabe in jeglichem Medium und Format erlaubt, sofern Sie den/die ursprünglichen Autor(en) und die Quelle ordnungsgemäß nennen, einen Link zur Creative Commons Lizenz beifügen und angeben, ob Änderungen vorgenommen wurden.

Die in diesem Artikel enthaltenen Bilder und sonstiges Drittmaterial unterliegen ebenfalls der genannten Creative Commons Lizenz, sofern sich aus der Abbildungslegende nichts anderes ergibt. Sofern das betreffende Material nicht unter der genannten Creative Commons Lizenz steht und die betreffende Handlung nicht nach gesetzlichen Vorschriften erlaubt ist, ist für die oben aufgeführten Weiterverwendungen des Materials die Einwilligung des jeweiligen Rechteinhabers einzuholen.

Weitere Details zur Lizenz entnehmen Sie bitte der Lizenzinformation auf http://creativecommons.org/ licenses/by/4.0/deed.de.

\section{Literatur}

1. Brown CJ, Mutran EJ, Sloane PD et al (1998) Primary care physicians' knowledge and behavior related to Alzheimer's disease. J Appl Gerontol 17(4):462-479

2. Casey A-N, Islam M, Schütze H et al (2020) GP awareness, practice, knowledge and confidence: evaluation of the first nation-wide dementiafocused continuing medical education program in Australia. BMCFam Pract 21:104

3. Connell CM, Boise L, Stuckey JC et al (2004) Attitudes toward the diagnosis and disclosure of dementia among family caregivers and primary care physicians. Gerontologist 44(4):500-507

4. Deutsche Gesellschaft für Psychiatrie und Psychotherapie, Psychosomatik und Nervenheilkunde (DGPPN), Deutsche Gesellschaft für Neurologie (DGN) S3-Leitlinie „Demenzen“. https://www. awmf.org/uploads/tx_szleitlinien/038-013I_S3Demenzen-2016-07.pdf. Zugegriffen: 27. Sept. 2020

5. Donath C,GräßelE, Großfeld-Schmitz Metal (2010) Effects of general practitioner training and family support services on the care of home-dwelling dementia patients - results of a controlled clusterrandomized study. BMC Health Serv Res 10:314

6. Eichler T, Thyrian JR, Hertel J et al (2014) Rates of formal diagnosis in people screened positive for dementia in primary care: results of the Delphitrial. J Alzheimers Dis 42(2):451-458

7. Geschke K, Scheurich A, Schermuly I et al (2012) Effectivity of early psychosocial counselling for family caregivers in general practitioner based dementia care. Dtsch Med Wochenschr 137(43):2201-2206

8. Hirsch RD (2008) Im Spannungsfeld zwischen Medizin, Pflege und Politik: Menschen mit Demenz.ZGerontol Geriat41(2):106-116

9. Kurz A, Pohl C, Ramsenthaler M, Sorg C (2009) Cognitive rehabilitation in patients with mild cognitive impairment. Int J Geriatr Psychiatry 24(2):163-168
10. Landeskrankenhaus (2020) DemStepCare. https://www.landeskrankenhaus.de/ aktuelles/projekte-im-landeskrankenhaus/ zentrum-fuer-psychische-gesundheit-imalter-zpga/demstepcare-das-hausarztbasierteversorgungskonzept. Zugegriffen: 23. Dez. 2020

11. Laux N, Melchinger H, Scheurich A et al (2010) Verbesserte ambulante Demenzversorgung. Das hausarztbasierte rheinland-pfälzische Modellprojekt ,start-modem'. Dtsch Med Wochenschr 135(44):2175-2180

12. Linden M, Horgas AL, Gilberg R et al (1997) Predicting health care utilization in the very old: the role of physical health, mental health, attitudinal and social factors. J Aging Health 9(1):3-27

13. Löppönen M, Raiha I, Isoaho R et al (2003) Diagnosing cognitive impairment and dementia in primary health care - a more active approach is needed. Age Ageing 32(6):606-612

14. Low L-F, McGrath M, Swaffer K, Brodaty H (2019) Communicating a diagnosis of dementia: a systematic mixed studies review of attitudes and practices of health practitioners. Dementia 18(78):2856-2905

15. Mansfield E, Noble N, Sanson-Fisher R, Mazza D, Bryant J (2019) Primary care physicians' perceived barriers to optimal dementia care: a systematic review. Gerontologist 59(6):697-708

16. Mayring $P$ (2010) Qualitative Inhaltsanalyse. Grundlagen und Techniken, 11. Aufl. Beltz, Weinheim

17. Melchinger $\mathrm{H}$, Machleidt W (2005) Hausärztliche Versorgung von Demenzkranken. Analyse der IstSituation und Ansätze für Qualifizierungsmaßnahmen. Nervenheilkunde 6:493-498

18. Mißlbeck A (2009) Plädoyer für gemeinsame Behandlungspfade. https://www.aerztezeitung.de/ Politik/Demenz-Plaedoyer-fuer-gemeinsameBehandlungspfade-372175.html. Zugegriffen: 23 . Dez. 2020

19. Pentzek M, Vollmar HC, Wilm S, Leve V (2017) Putting dementia awareness into general practice: the CADIF approach. Z Gerontol Geriatr 50(2):44-47

20. Pimlott NJ, Persaud M, Drummond N et al (2009) Family physicians and dementia in Canada: part 1 Clinical practice guidelines: awareness, attitudes, and opinions. Can Fam Physician 55(5):506-507.e5

21. Radisch J, Baumgardt J, Touil E, Moock J, KawohIW (2015) Demenz - Behandlungspfade für die ambulante integrierte Versorgung. Kohlhammer, Stuttgart

22. Reuster T, Jurjanz L, Schützwohl M et al (2008) Effektivität einer optimierten Ergotherapie bei Demenz im hausärztlichen Setting. Z Gerontol Geriatr 21(3):185-189

23. Romero B (2004) Selbsterhaltungstherapie: Konzepte, klinische Praxis und bisherige Ergebnisse. ZGerontol Geriatr 17(2):119-134

24. Schencking M, Keyser M (2007) Demenz - Was kann der Hausarzt tun? - Soll-Ist-Vergleich in der Versorgung demenzkranker Patienten. Notf Hausarztmed 33(12):576-578

25. Schers HJ, van den Hoogen $\mathrm{H}$, Bor $\mathrm{H}$ et al (2005) Familiarity with a GP and patients' evaluations of care. A cross-sectional study. Fam Pract 22(1):15-19

26. Stoppe G (2011) Demenz: Frühdiagnose und ambulante Versorgung. In: Stoppe G (Hrsg) Die Versorgung psychisch kranker alter Menschen. Bestandsaufnahme und Herausforderung für die Versorgungsforschung. Deutscher Ärzteverlag, Köln, S 133-140
27. Tang EYH, Birdi R, Robinson L (2018) Attitudes to diagnosis and management in dementia care: views of future general practitioners. Int Psychogeriatr 30(3):425-430

28. Thyrian JR, Eichler T, Michalowsky B et al (2016) Community-dwelling people screened positive for dementia in primary care: a comprehensive, multivariate descriptive analysis using data from the Delphi-study. J Alzheimers Dis 52(2):609-617

29. Thyrian JR, Fiss T, Dreier A et al (2012) Life- and person-centred help in Mecklenburg-Western Pomerania, Germany (DelpHi): study protocol for a randomised controlled trial. Trials 13(1):56

30. Wangler J, JanskyM(2020) Dementia diagnostics in general practitioner care. Do general practitioners have reservations? - the findings of a qualitative study in Germany. Wien Med Wochenschr 170(910):230-237

31. Wangler J, Fellgiebel A, Jansky M (2018) Hausärztliche Demenzdiagnostik - Einstellungen, Vorgehensweisen und Herausforderungen von Hausärzten in Rheinland-Pfalz. Dtsch Med Wochenschr 143(19):165-171

32. Wangler J, Fellgiebel A, Mattlinger C et al (2018) Diagnostik und Versorgung der Demenz - eine Herausforderung für die Hausarztmedizin. Z Allg Med 94(1):12-16

33. Wolf D, Rhein C, Geschke K, Fellgiebel A (2019) Preventable hospitalizations among older patients with cognitive impairments and dementia. Int Psychogeriatr 31(3):383-391

34. Wollny $A$, Fuchs $A$, in der Schmitten $J$ et al (2010) Zwischen Nähe und Distanz. Eine Studie zu hausärztlichen Wahrnehmungsweisen von an Demenz erkrankten Personen. Z Allg Med 86(11):425-434

35. Yaffe MJ, Orzeck P, Barylak L (2008) Family physicians' perspectives on care of dementia patients and family caregivers. Can Fam Physician 54(7):1008-1015 